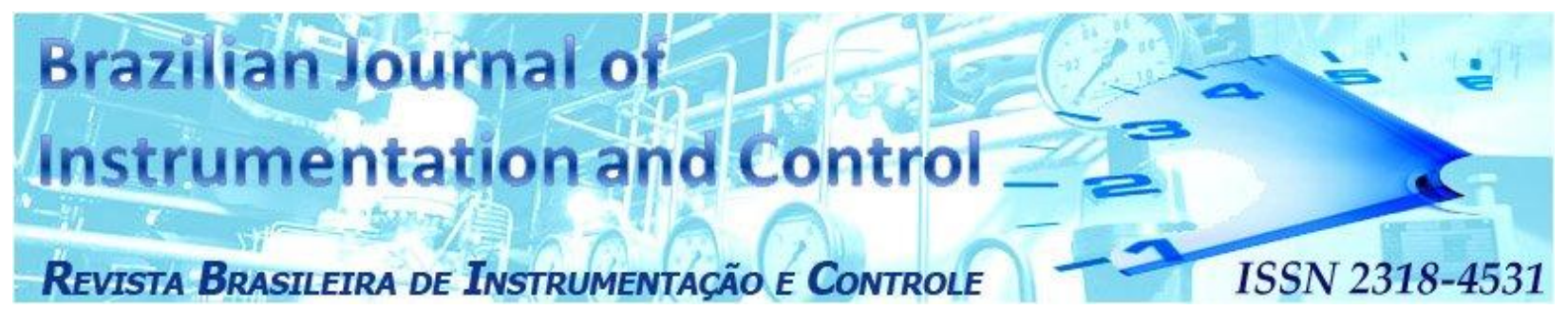

\title{
Leveraging Wireless Devices for Networked Control Systems in Industrial Applications
}

\author{
Eduardo P. Godoy, Thiago A. Oliveira, Fernando Scorzoni and Arthur J.V. Porto
}

\begin{abstract}
Networked control systems (NCSs) are distributed control system in which sensors, actuators and controllers are physically separated and connected through communication networks. NCS represent the evolution of networked control architectures providing greater modularity and control decentralization, ease maintenance and diagnosis and lower cost of implementation. A recent trend in this research topic is the development of NCS using wireless networks (WNCS) which enable interoperability between existing wired and wireless systems. This paper presents the feasibility analysis of using serial to wireless converter as a wireless sensor link in NCS. In order to support this investigation, relevant performance metrics for wireless control applications such as jitter, time delay and messages lost are highlighted and calculated to evaluate the wireless converter capabilities. In addition the control performance of an implemented motor control system using the converter is analyzed. Experimental results led to the conclusion that serial ZigBee device is recommended against the Bluetooth as it provided better metrics for control applications. However, both devices can be used to implement WNCS providing transmission rates and closed control loop times which are acceptable for NCS applications. Moreover the use of the wireless device delay in the PID controller discretization can improve the control performance of the system.
\end{abstract}

Index Terms-Networked Control Systems, ZigBee, Bluetooth, Wireless Performance Metrics

\section{INTRODUCTION}

$\mathrm{N}$ etworked Control Systems (NCSs) have become a widely researched topic due to the challenges and opportunities of developing a system which merges knowledge of three fields: control theory, communication networks and real-time

Eduardo Paciência Godoy (corresponding author) and Thiago de Almeida Oliveira are with the São Paulo State University (UNESP), Sorocaba, São Paulo, Brazil, e-mail: epgodoy@ sorocaba.unesp.br.

Fernando Scorzoni and Arthur Jose Vieira Porto are with the University of São Paulo (EESC-USP), São Carlos, São Paulo, Brazil, e-mail: fernandosc@yahoo.com, ajvporto@sc.usp.br. systems [1]. The introduction of NCS can improve the efficiency, flexibility and reliability of distributed control system in industrial applications through reduced wiring and distributed intelligence with consequent reduction in the installation, reconfiguration and maintenance [2].

The NCS technology differs from the traditional fieldbus systems in that the controller and the plant, sensors and actuators are physically separated and connected through an industrial network [1]. As can be seen in Fig. 1, the control signal is sent to the controller by a message transmitted over the network while the sensor samples the plant output and returns the information to the controller also by transmitting a message over the network.

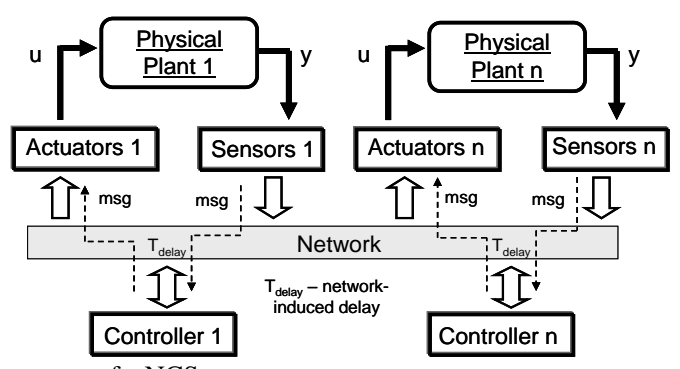

Fig. 1. Structure of a NCS

Even though the NCS offers many advantages over traditional centralized control systems, several challenges also emerged giving rise to many important research focuses. New control strategies to deal with jitter, delays and packet losses, reliability and security of communications, bandwidth allocation, development of data communication protocols, design of fault detection and fault tolerant systems, real-time information collection and scheduling are some of the relative topics extensive studied [3]. Another important parameter that affects the NCS performance and stability is the messages sampling interval [4]. The NCS must sample and transmit data into messages at a sampling interval appropriate to achieve required performance metrics. However, if this sampling 
interval is higher than the network bandwidth available, the network becomes overloaded, originating additional network delays and jitter, and causing packet losses and errors in the transmissions of messages [5].

Recent advances in wireless sensor networking technology have led to the development of low cost, low power, multifunctional sensor nodes. With these advances, a new trend has emerged in using wireless networks in NCS [6]. These systems are known as Wireless Networked Control Systems (WNCS). WNCS have lately been attracting many research efforts driven by the increasing developments and standardization such as Bluetooth, ZigBee and WiFi [7]. For the latter, its high power consumption and the short autonomy of a battery power supply are considered an important disadvantage.

Many interesting characteristics inherent to wireless networks are motivating the development of WNCS [8]. For fieldbus control systems, there is always the risk of cutting the bus that connects all the devices. NCS using wireless networks can eliminate all the problems arising from wires in the system. This is the most important advantage of using such technology. WNCS can operate in a wide range of environments, enabling interoperability between existing wired and wireless systems and also providing advantages in power and flexibility when compared to wired ones [6]. Another advantage for WNCS is the feasibility of installation in places where cabling is impossible, such as sensors in hazardous ambient and plants with intrinsic security zones.

A common architecture for WNCS includes a plant in which the sensor and the controller have a point to point wireless communication between them. It is easy to understand why to first migrate the sensor link to a wireless connection. As the actuator usually needs relative high power to drive the plant, sometimes it is not reasonable to implement a wireless connection to this link if the actuator will continue requiring a physical connection for power supply or even if the actuator lifetime will be deeply decreased in this situation [7]. To provide wireless remote monitoring capabilities for industrial controllers such as PLCs, a simple solution that has been applied is the use of serial to wireless devices. These devices are electronic interfaces which receive data from serial connections, quite common in industrial devices, and retransmit them through wireless connections.

This paper aims to leverage wireless devices for NCSs in industrial applications. The main idea is to investigate the feasibility of using serial to wireless converters (ZigBee and Bluetooth) to implement WNCS. To support this investigation, wireless performance metrics such as jitter and time delay have been measured to evaluate the device capabilities and also the performance of an implemented motor control system has been analyzed. Experimental results led to the conclusion that the serial ZigBee device provided better performance metrics than the Bluetooth, even though both could be used to implement WNCS providing transmission rates and closed control loop times which are acceptable for NCS.

\section{Control using Wireless NetWorks}

According to [6], the most common application of wireless networks is for monitoring purposes which requires reliability in the transmission of messages. The reliability is quite related to the packet losses or even messages not corrected transmitted on the network. Typically, packet losses result from transmission errors in physical network links or from buffer overflows due to congestion. Additionally, in monitoring applications there is no hard time constraints on these messages deliveries since the sampling interval is usually given in seconds to ensure a great battery lifetime [7].

On the other hand, when wireless networks are used for control applications, such as in WNCS, additional factors need to be considered. Contrary to monitoring applications, WNCS do not need to maximize the reliability. Reference [9] presents some results on developing NCS to overcome the packet losses effects on its performance and stability. Among the additional factors that need to be considered for WNCS implementation, [10] indicates the time delay, the jitter, realtime capabilities and the limited bandwidth.

The transmission of information (sensor data and control signal) in a NCS or WNCS needs to be done using messages over a network. The overall time delays between sampling the sensor and the actuation on the plant can be high variable because, for example, network delays, encoding and decoding times and transmission delays on the used network (wired or wireless). Reference [11] presents a detailed discussion about the components of this time delay in NCS. Consequently, if time constraints are not met, which means that the expected time was affected by the delays, the correct execution of the designed control may be compromised, thus making the NCS more oscillatory and even unstable [3].

Generally in NCS, jitter can be defined as a variation in the time delay of consecutive network messages transmitted and can be measured by the deviation of the transmission time in network messages. Reference [2] affirms that usually the jitter problem is tied to the hardware used and software coding (algorithm and scheduling). Investigating the jitter in WNCS is important to ensure that the message transmission delays will be kept between predefined maximum and minimum values and this variability will not affect the controller period and thus degrade the system performance.

According to [8], a key performance element for a WNCS is the capability to support real-time applications. To correct understand this term for NCS, real-time means that the system must be able to response to control requests timely, so that corrections still have their desired effect on process operation. Resuming, for WNCS design a tradeoff between time delays, packet losses and jitter will be required to provide deterministic operation and achieve control and stability requirements.

\section{WNCS RESEARCH PLATFORM}

\section{A. Experimental Setup}

The platform used for the experiments in this paper has been developed for NCS and WNCS research and 
experimentation [12], [13]. The communication network used for the NCS integration (sensor data acquisition and transmission of the control signal to the actuator) was the CAN protocol. In this paper the use of a serial to ZigBee and a serial to Bluetooth devices as the sensor communication links, thus implementing a WNCS, are evaluated. However, the actuator link will continue using the CAN network.

The implementation structure shown in Fig. 2 is related to one NCS of the platform. The defined motor velocity control system has two microcontroller-based electronic control units (ECU), in which one is responsible for the sensor data acquisition and transmission of the information in the Bluetooth and ZigBee wireless networks, and the other is responsible for the actuation in the plant and communication with the CAN-based network. A desktop with LabVIEW, a PCI-CAN interface and a serial Bluetooth and ZigBee converters were used for the development of the control methodologies for NCS.

\section{DESKTOP CONTROLLER}
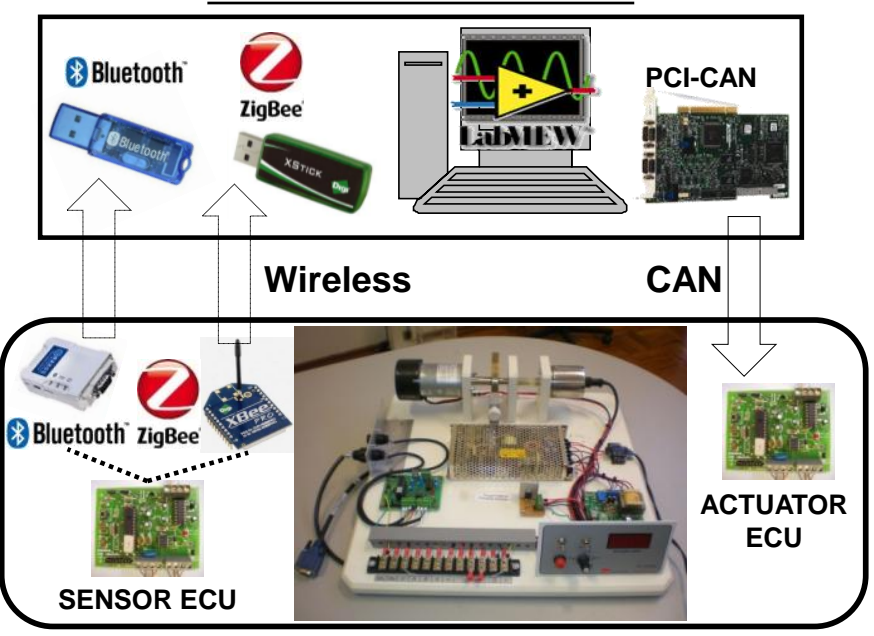

CLOSED LOOP - VELOCITY CONTROL

Fig. 2. Implementation Structure: Adding Wireless Networks to the NCS Research Platform

Considering the Bluetooth, in the experimental setup the controller is the master and the sensor is a slave. It provides the capability of one controller to receive (and controls) information from up to 7 sensors, defined in a Bluetooth Piconet. The time-driven sensor node samples the plant or process periodically and sends the information to the controller node over the Bluetooth network. Upon receiving a sample, the controller computes a control signal which is sent through the CAN network to the actuator node, where it is subsequently actuated. The threads executing in the controller and actuator nodes are both event-driven, which means that their actions is performed as soon as they receive messages.

Considering the ZigBee network, in the experimental setup the controller is configured as Coordinator and the sensor as a Router or End Device, with point to point communication (no hops on the message transmission). It provides a multicast transmission or the capability of one controller to receive (and control) information from more than one sensor. The timedriven sensor node samples the plant periodically and sends the information to the controller node over the ZigBee network. Upon receiving a sample, the controller computes a control signal which is sent through the CAN network to the actuator node, where it is subsequently actuated. The threads executing in the controller and actuator nodes are both eventdriven, which means that their actions is performed as soon as they receive messages.

\section{B. Overview of Communication Networks}

The CAN protocol [14] has been previously used as the communication network is due to its low cost of development and large acceptance in the industrial and academic areas. The CAN has been developed to interconnect ECUs in automotive area, but recently it has also been applied in many other networked applications. Currently, CAN-based NCS are applied as a solution for distributed systems in several areas, such as robotic, automated manufacturing and process control, and they are also used in proprietary architectures such as Device Net and CAN Open.

As described in [15], in CAN-based networks data are transmitted and received using messages that carry data from a transmitting node to one or more receiving nodes. An identifier, unique throughout the network, labels each message and its value defines the priority of the message to access the network. The CAN protocol is optimized for short messages and uses a CSMA/CD with NDBA (Carrier Sense Multiple Access / Collision Detection with Non-Destructive Bitwise Arbitration) arbitration access method. The bit stream of a transmission is synchronized on the start bit, and the arbitration is performed on the following message identifier, in which a logic zero is dominant over a logic one.

Bluetooth (also known as the IEEE 802.15.1 protocol) was developed as a wireless protocol for short-range communication in wireless personal area networks (PAN) as a cable replacement for mobile devices. The Bluetooth technology utilizes the unlicensed ISM band and its maximal data rate is $1 \mathrm{Mb} / \mathrm{s}$, but the useful data rate is much lower. The typical power limit for the Bluetooth system is $4 \mathrm{dBm}$ with a nominal range of 10 meters. Bluetooth is mainly designed to maximize ad hoc networking functionality [16].

The Bluetooth system adopts a frequency hopping spreadspectrum (FHSS) communication, over 79 carriers of $1 \mathrm{MHz}$ bandwidth between $2402 \mathrm{MHz}$ and $2480 \mathrm{MHz}$, which transmits data over different frequencies at different time intervals. During operation, the system hops from one carrier to the next in a pseudo-random sequence at 1600 hops per second, resulting in a slot time of $625 \mu$ s at each carrier. This gives relatively short packet length and good interference protection [7]. Bluetooth uses a master-slave-based MAC (medium access control) protocol and up to eight Bluetooth units may be interconnected in a so called Piconet. A packet is sent at each hop (it is possible for a data unit to be spread over 5 hops) and an acknowledgment is received. In the case of a malformed transmission or a lost link, the MAC layer executes a retry. 
The IEEE 802.15.4 standard defines the protocol and interconnection of devices via radio communication in a low data rate, low power consumption, and low cost personal area network (PAN). ZigBee builds upon the IEEE 802.15.4 standard which defines the physical and data layers [17]. ZigBee standardizes the higher layers of the protocol stack. The network layer is in charge of organizing and providing routing over a multi hop network (built on top of the IEEE 802.15.4 functionalities), while the Application Layer intends to provide a framework for distributed application development and communication. ZigBee network layer defines three device types: End device (simple device), Router (routing capabilities device) and Coordinator (managing device).

The physical layer provides an interface between the MAC sub layer and the physical radio channel. It usually defines a frequency bands of 2,4Ghz (with 16 channels) using the Direct Sequence Spread Spectrum (DSSS) access mode with a data rate of $250 \mathrm{Kbps}$. The data layer provides an interface between upper layers and the physical layer. It handles channel access, link management, frame validation, security, and nodes synchronization [17]. The MAC protocol supports two operational modes that may be selected by the coordinator: beacon enabled mode and non-beacon enabled mode. In the non-beacon enabled mode the MAC is provided by an unslotted CSMA/CA (Carrier Sense Multiple Access/ Collision Avoidance) mechanism. In the beacon enabled mode, the beacons are periodically sent by ZigBee coordinator to synchronize nodes that are associated with it, and to identify the PAN.

\section{FEASIBILITY ANALYSIS AND EXPERIMENTAL RESULTS}

\section{A. Wireless Networks Performance Metrics}

There are several network performance metrics utilized in the evaluation and analysis of distributed control systems using networks. According to [5], the principal metrics that characterize the performance of NCS are average time delay and jitter. The most critical of these metrics for the achievable NCS closed loop sample rate is the time that elapses between sending data at one node and receiving it at the other or the time delay. It is hard to experimentally measure the time delay taken by a node to process a message and transmit it. Consequently the only way to measure this time delay is from roundtrip communication time within the loop. The round trip time is the time delay in sending a packet from one node to another and back. And the same is true if using a serial to wireless device that receives some data from a RS232 connection and retransmits it through the ZigBee or the Bluetooth. If a statistical calculation is performed from an amount of collected time delays, we can obtain the average time delay (Td) from the transmissions of messages in the wireless network using the device.

The variability in measured values of the time delay will give the jitter (J) in the developed WNCS. Depending on the jitter (the Standard Deviation of the values), the NCS performance can be significantly affected because it will be impossible to calculate a reliable closed loop sampling rate for the system. The worst value of the time delays distribution will give the minimum value that could be used for the NCS controls loop using the serial to wireless device. However, as we are using the serial to wireless device only as the sensor link in the NCS, the half of the average time delay (equal to $\mathrm{Td} / 2$ ) and the half of the jitter (equal to $\mathrm{J} / \sqrt{ } 2$ ) will be used as the performance metrics in this paper.

The experiments have been carried out with ambient noise and other traffic on the ZigBee and Bluetooth channels. Two additional serial ZigBee and serial Bluetooth devices on the same network have been connected to increase the transmission of messages and share the bandwidth. Moreover, ambient noise from cordless phones using $2.4 \mathrm{GHz}$ frequency band can also affect the transmissions. Even though these phenomena are not quantified, both are considered in the experiment. The performance analysis experiments used a 1000 messages transmission cycle with a 2 bytes data length (minimum 16 bits variables used in the control experiments) and the maximum baud rate $115200 \mathrm{bps}$ available at the ECU and at the serial ZigBee and serial Bluetooth devices.

Firstly the serial ZigBee device was evaluated [18]. Fig. 3 shows the distribution of the time delays obtained with the roundtrip test using the serial RS232 ZigBee device with a 10 $\mathrm{m}$ distance. As can be seen the majority of values are close to the mean value of $27,38 \mathrm{~ms}$ without presence of large outliers, which means a good determinism.

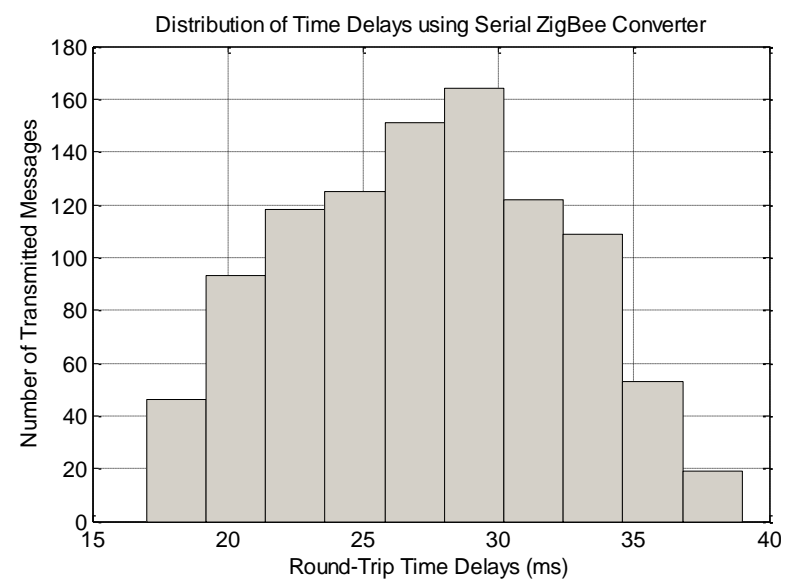

Fig. 3. Histogram of the Average Time Delay with the Serial ZigBee Device at $10 \mathrm{~m}$

Table I presents a resume of the performance metrics for the serial ZigBee device calculated for different distances of 5, 10 and $20 \mathrm{~m}$.

TABLE I

Performance Metrics with the Serial ZigBee Device at DIFFERENT DISTANCES

\begin{tabular}{ccccc}
\hline $\begin{array}{c}\text { Distance } \\
(\mathrm{m})\end{array}$ & $\begin{array}{c}\mathrm{Td} / 2 \\
(\mathrm{~ms})\end{array}$ & $\begin{array}{c}\mathrm{J} \sqrt{ } 2 \\
(\mathrm{~ms})\end{array}$ & $\begin{array}{c}\text { Worst Value } \\
(\mathrm{ms})\end{array}$ & $\begin{array}{c}\text { Message Loss } \\
(\%)\end{array}$ \\
\hline 5 & 14,22 & 2,5 & 22,5 & 0 \\
\hline 10 & 14 & 2,46 & 19,51 & 0 \\
\hline 20 & 14,12 & 2,48 & 21,6 & 0 \\
\hline
\end{tabular}


In accordance with the data in Table I, the average time delays in using the serial ZigBee device as the sensor link is around $14,1 \mathrm{~ms}$ with $2,5 \mathrm{~ms}$ of jitter (roundtrip time delay of $28,2 \mathrm{~ms}$ and $5 \mathrm{~ms}$ of jitter). There has been no significant difference in terms of time delay and jitter related to the increase in the distance tested. And for the distance tested of up to $20 \mathrm{~m}$, no message loss occurred proving the good reliability of the ZigBee network.

After that the serial Bluetooth device was also evaluated [19]. Fig. 4 shows the distribution of the time delays obtained with the round-trip test using the serial RS232 Bluetooth converter with a $10 \mathrm{~m}$ distance. As can be seen the majority of values are close to the average value (Td) of $75,62 \mathrm{~ms}$ without presence of large outliers.

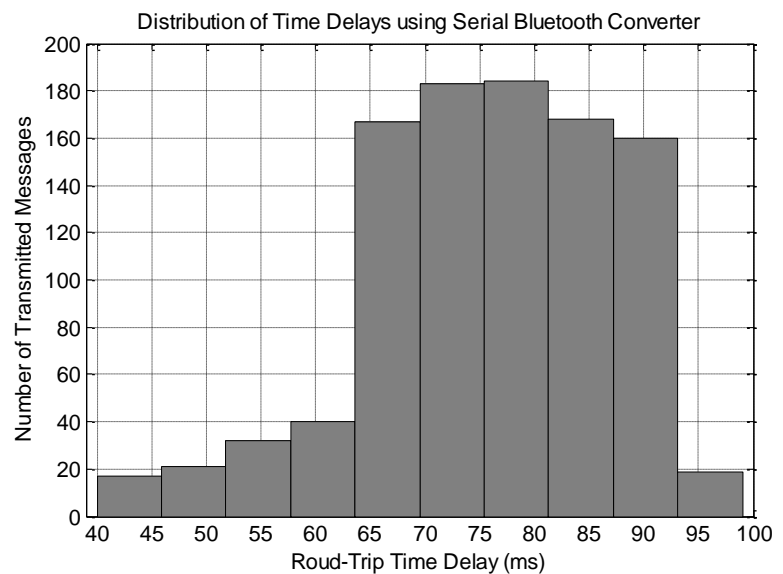

Fig. 4. Histogram of the Average Time Delay with the Serial Bluetooth Device at $10 \mathrm{~m}$

Table II presents a summary of the performance metrics for the serial Bluetooth converter as a sensor link, calculated for different distances of 5,10 and $20 \mathrm{~m}$.

TABLE II

Performance Metrics with the Serial Bluetooth Converter at Different Distances

\begin{tabular}{ccccc}
\hline $\begin{array}{c}\text { Distance } \\
(\mathrm{m})\end{array}$ & $\begin{array}{c}\mathrm{Td} / 2 \\
(\mathrm{~ms})\end{array}$ & $\begin{array}{c}\mathrm{J} / \sqrt{2} \\
(\mathrm{~ms})\end{array}$ & $\begin{array}{c}\text { Worst Value } \\
(\mathrm{ms})\end{array}$ & $\begin{array}{c}\text { Message Loss } \\
(\%)\end{array}$ \\
\hline 5 & 37,80 & 7,40 & 49,5 & $0,9 \%$ \\
\hline 10 & 37,81 & 7,68 & 49,5 & 1,2 \\
\hline 20 & 38,33 & 9,02 & 50 & 4,6 \\
\hline
\end{tabular}

In accordance with the data in Table II, the average time delays in using the serial Bluetooth converter as the sensor link is around $38 \mathrm{~ms}$ with $8 \mathrm{~ms}$ of jitter (half of the round-trip time delay of $76 \mathrm{~ms}$ and $11 \mathrm{~ms}$ of jitter). There has been no significant difference in terms of time delay and jitter related to the increase in the distance tested. On the other hand, the percentage of message lost grew from 0,9 to $4,6 \%$.

Comparing the results in Table I and II, and using the principal metrics that characterize the performance of NCS which are the average time delay and jitter [5], it can be concluded that the serial ZigBee device is the most adequate or recommended to be used for WNCS applications. The performance metrics obtained with the serial ZigBee are more than $100 \%$ better than the results of the serial Bluetooth. In addition, the serial ZigBee device has not shown message loss during the experiments carried out.

Reference [20] presents a performance evaluation of built-in ZigBee devices for electricity meters. The results of this paper showed a roundtrip delay of $18 \mathrm{~ms}$ with $2,5 \mathrm{~ms}$ of jitter, which are smaller (almost half of the values) than the obtained when using the serial ZigBee device. As a result, it is quite clear the device adds a significant time delay in the transmission of messages in the ZigBee. This additional delay is due to the required communication along the RS232 between the device hardware and the computer or ECU hardware and also the time necessary for codification between the RS232 and ZigBee standard. The jitter increase is related to the variability imposed by the presence of buffers in the device and the ZigBee protocol stack processing time. Even though the results of the built-in ZigBee devices are better, it is still important to evaluate the use of serial ZigBee devices for control applications as the built-in devices are more expensive and difficult to be found in industrial equipment.

In order to do that, the worst value of time delays obtained for the serial to wireless device can be used to define the maximum closed loop cycle time achievable for the WNCS when using it. Using the worst value of time delays in Table I that is equal to $22,5 \mathrm{~ms}$, the bottleneck in terms of how fast the closed control loop time could be is defined by the minimum value of $50 \mathrm{~ms}$. The minimum closed loop time of $50 \mathrm{~ms}$ is a reliable value as it must be at least less than the sum of the sensor transmission time $(22,5 \mathrm{~ms}$ worst case when using the device), the control algorithm computation time, the control signal transmission time (using CAN) and actuation on the plant. For this paper, a $100 \mathrm{~ms}$ value is defined for our WNCS implementation in which only the sensor uses the serial ZigBee device (the actuator uses CAN). Based on the conclusions of a previous author's paper [4], in which has been investigated that a $200 \mathrm{~ms}$ closed control loop is satisfactory to achieve an acceptable performance for the velocity control NCS, we can affirm that the serial ZigBee device can be used as the sensor link in the developed WNCS [18]. Moreover, the results can also demonstrate that the serial ZigBee device is feasible for any fully WNCS application (connecting the sensor and the actuator to the controller) with control loops greater than $100 \mathrm{~ms}$.

In the same manner for the serial Bluetooth device, using the worst value of time delays in Table II that is equal to $50 \mathrm{~ms}$, the bottleneck of closed control loop time is defined by the minimum value of $100 \mathrm{~ms}$. Based on the same previous conclusion, even though the serial Bluetooth provided a worse performance in comparison with the serial ZigBee, we can affirm that it can also be used as the sensor link in the developed WNCS [19]. Moreover, the results can also demonstrate that the serial Bluetooth converter is feasible for any fully WNCS application (connecting the sensor and the actuator to the controller) with control loops greater than $150 \mathrm{~ms}$. 


\section{CONTROL DESIGN AND PERFormance COMPARISON}

To prove that the serial to wireless devices could be used for WNCS as affirmed in the last section, the structure shown in Fig. 2 was used for control tests and experimentation. According to [21], controllers for NCS cannot be designed with continuous time control theory because the resulting performance is unsatisfactory. The controllers for NCS have to handle the network delay effects in the systems and need to be designed in discrete time.

The PID controller developed in [21] was used for the WNCS application. This controller is a discrete time PID derived with the backward derivative approximation with setpoint weighting, reference off, filtering on derivative part and Anti-Windup of the integrative part. Fig. 5 presents the structure of the discrete PID designed for the NCS of the platform.

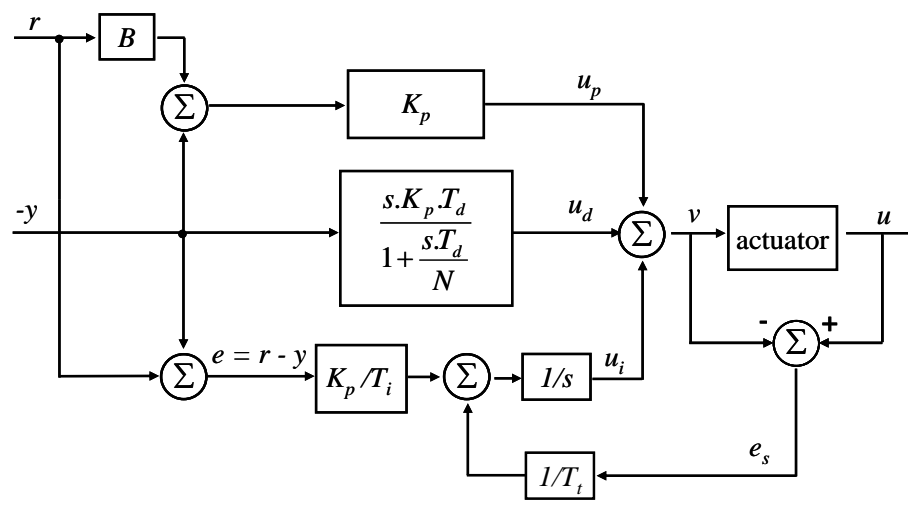

Fig. 5. Block Diagram of the PID Controller Used

For the WNCS operation, the following parameters were used: CAN speed of $250 \mathrm{kbit} / \mathrm{s}$ and messages data length of two bytes for all ECUs, sensor sampling interval (discretization time) of $100 \mathrm{~ms}, \mathrm{~N}=10$ in the constant filtering, $\mathrm{B}=1$ in the reference weighting constant and $\mathrm{Tt}=\mathrm{Ti}$ for PI controllers.

The first results of the closed loop WNCS for the motor velocity control is shown in Fig. 6. The idea of this graph is to compare the performance of the NCS only using CAN (previously) with the performance using the serial to wireless devices (ZigBee and Bluetooth) thus implementing a WNCS.

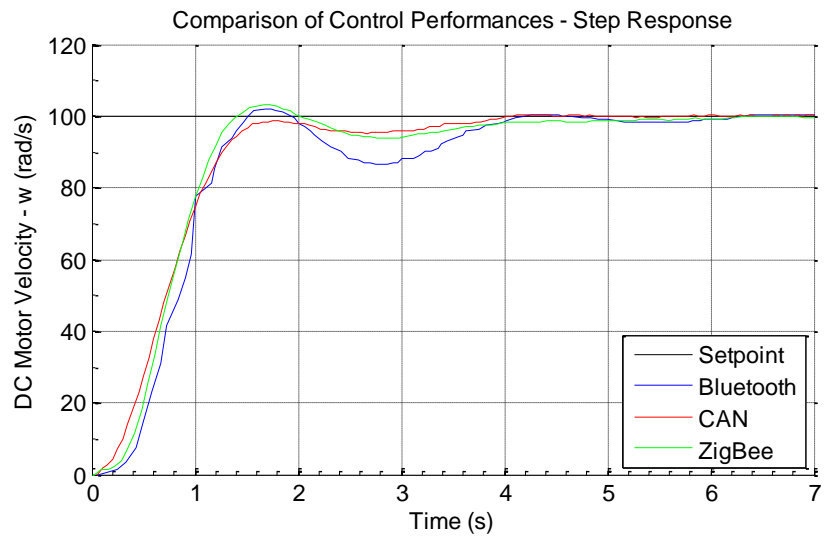

Fig. 6. Comparison of Control Performances: CAN-based NCS, ZigBee WNCS and Bluetooth WNCS
To make the comparison in Fig. 6, the PID parameters configured using the Ziegler/Nichols method in the CAN test $(\mathrm{P}=0,07$ and $\mathrm{Ti}=0,65)$ were also used in the WNCS implementations. The graph of Fig. 6 shows the comparison of the closed loop control performances between the implementation of the NCS using only the CAN network and with the ZigBee and Bluetooth networks as the sensor links. The large delays and jitter of using the serial to wireless devices as the network degraded the performance of the system. Comparing the curves, an increase in the response time (related to the greater value of the delay in the Bluetooth) can be seen as well as the output becomes a little more oscillatory. This graph proves that both serial to ZigBee and to Bluetooth can be used for the WNCS, but as concluded the performance for the case using the ZigBee device is better. As the serial ZigBee device is the recommended for use, the following experiments used only this device.

It's important to verify that the discrete time PID controller used in the experiments uses a discretization time of $100 \mathrm{~ms}$, which is equal to the sensor sampling interval. However as we are using the serial ZigBee device as the sensor link, the greater values of this device time delay and jitter will impact (and increase) this fixed value of $100 \mathrm{~ms}$ and consequently degrade the WNCS control performance.

A common approach to handle the network delay effect in NCS is to include this information on the controller discretization. Usually it can be done in two ways. The best way is measuring (with time stamps) or estimating the time delays online and dynamically correcting the discretization parameter during the NCS operation. A simpler method adds the time delay information collected from experiments to the discretization time, making it more realistic.

The time delay information obtained from the serial ZigBee device evaluation experiment can be therefore used in the PID controller discretization. This information can be obtained by a normal distribution of the delays presented in Fig. 3, resulting in the Fig. 7.

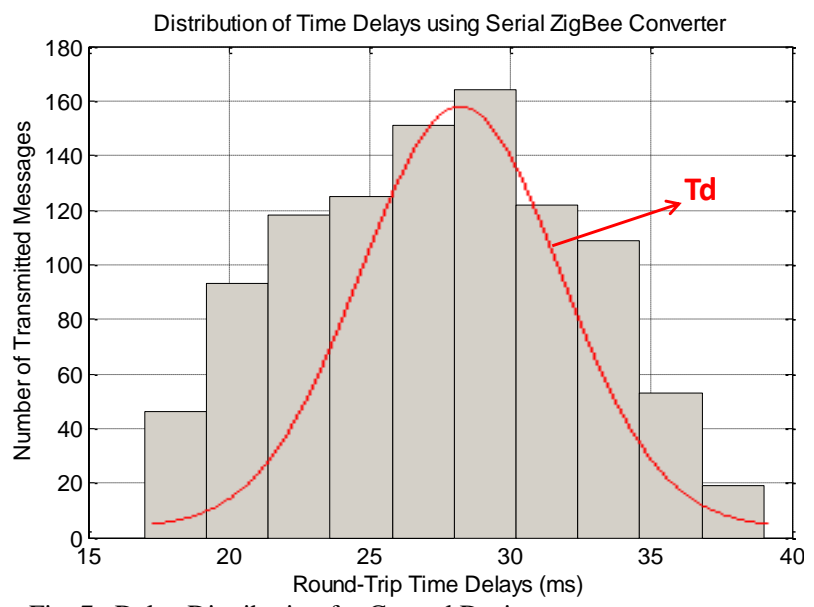

Fig. 7. Delay Distribution for Control Design

As a result the discretization parameter $(d)$ shown in (1) is used, in which $h$ is the sensor sampling interval and $T d / 2$ is the 
average time delay of the serial ZigBee device obtained by the delay distribution in Fig. 7.

$$
d=h+T d / 2
$$

In order to evaluate if there is an advantage in using the time delay information on the PID discretization, two control performance indexes were used for comparison. The Integral of the Absolute Error (IAE) performance index and a created Difference Index $(I d)$ in (2). This difference index calculates how different the process variable is from the desired output (SP - setpoint), during the total time of the experiment (T), giving a result in percentage (\%) used to determine which control system is better and how much better is it.

$$
I d=\frac{I A E}{\int_{0}^{T} S P(t) d t} x 100
$$

An experiment was defined to evaluate the application of delay information PID controller. An experiment time (T) of 30 s with a profile of different setpoints was used to compute the performance indexes. Fig. 8 presents the output responses of the WNCS under investigation using both cases: the fixed discrete time PID controller and the PID controller discretized with the time delay information. The PID parameters used in this experiment are equal for both controllers.

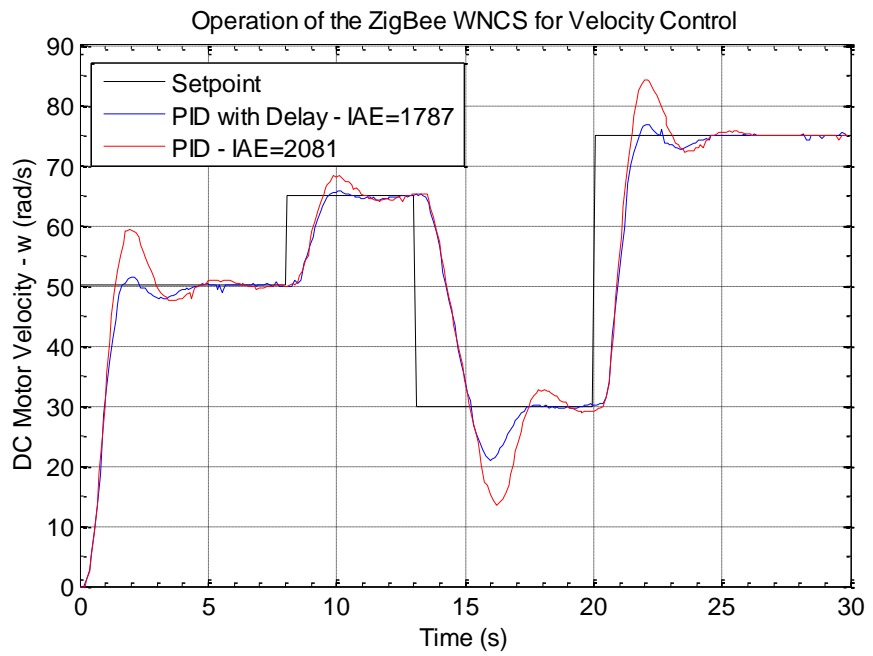

Fig. 8. Control Performance of the PID with Delay Information

TABLE III

Control Performance Metrics For the ExPERIMENT

\begin{tabular}{ccccc}
\hline Case study & $\begin{array}{c}\text { Overshoot } \\
(\%)\end{array}$ & $\begin{array}{c}\text { Settling } \\
\text { time }(\mathrm{s})\end{array}$ & $\begin{array}{c}\text { IAE } \\
\text { Index }\end{array}$ & $\begin{array}{c}\text { Id } \\
\text { Index } \\
(\%)\end{array}$ \\
\hline PID & 41,11 & 5,68 & 2081 & 12,42 \\
\hline $\begin{array}{c}\text { PID with } \\
\text { Delay } \\
\text { Measurement }\end{array}$ & 25,88 & 4,17 & 1787 & 10,65 \\
\hline
\end{tabular}

Comparing the graphs of the Fig. 8, it is quite clear that adding the delay information on the PID controller discretization provided an improvement in the control performance. The Table III presents the control performance metrics obtained with the experiment (worst-case overshoot and settling time). This improvement was mainly in terms of the reduction of the overshoot and oscillations (settling time) as can be seen in the Table III. This conclusion is also demonstrated by the difference in terms of the IAE index, which is equal to 1787 for the controller with the delay information and equal to 2081 using the basic PID controller.

Using the difference index (Id) created, it is possible to quantify how much was the advantage of using the delay information on the PID controller. The calculated difference indexes were 12,42\% for the basic PID controller and 10,65\% for the PID with the delay information. It is important to remember that this index computes the total error related to the difference between the controlled variable and the desired output given by the setpoint. Therefore, an advantage of almost $2 \%$ of the total control performance was obtained with the simple use of the PID controller with the delay information offline measured from the serial ZigBee device.

Even though the method applied in this paper of adding the time delay information on the PID discretization is not the best (if compared to the cases in which the delay is measured or estimated in each cycle of the control loop), it is simple and very easy to use and provide a better performance for the NCS.

Future work aims to develop a controller to estimate online the delays in the WNCS (improving the quality of the delay information), as a solution for the use of time stamps in wireless networks is still under research and development.

\section{CONCLUSION}

The increasing and widespread use of wireless sensor networks has driven the interest in researching and developing wireless networked control systems (WNCS). Aiming to provide wireless monitoring to already deployed networked systems, simple serial to wireless (Wi-Fi, Bluetooth and ZigBee) converters have been applied. However, these serial to wireless converters may also represent cheap (comparing devices with built-in wireless) and reliable solutions for control applications (instead of monitoring) using wireless networks, with the additional possibility to be used on traditional devices (sensors and actuators), which are currently available.

In this paper the use of serial to ZigBee and to Bluetooth devices for application as the sensor link in WNCSs were investigated. A revision about specific performance metrics commonly used to evaluate NCS using wireless networks was presented. Some of these performance metrics such as average time delay, jitter, worst case delay and messages lost were calculated to support the evaluation of the most adequate wireless network device to implement WNCS.

Based on the performance metrics results obtained from experiments, it can be concluded that the serial RS232 ZigBee 
device is recommended against the serial Bluetooth and can be used to implement WNCS providing transmission rates and closed control loop times that are acceptable for several networked control applications. For use only as the sensor link in WNCS, the device can provide reliable sampling intervals and closed control loops up to 50ms. Furthermore, if used in fully WNCS applications (connecting the sensor and the actuator to the controller) the serial ZigBee device must only be used with closed control loops greater than $100 \mathrm{~ms}$. Another conclusion is that the serial ZigBee devices must not be used for WNCS implementations with hard constraints on the response time, because the time delay related to the serial to wireless conversion is at least $10 \mathrm{~ms}$ in any message transmission.

The serial ZigBee device was used as a sensor link connection in a WNCS implementation of a motor velocity control, providing acceptable performance to the system with control closed loops of $100 \mathrm{~ms}$ and proving its feasibility for WNCS applications. Another important conclusion of this experiment is that adding the serial ZigBee device delay information, which can be easily offline measured from the device, in the controller discretization resulted in an improvement of $2 \%$ of the global control performance in the case studied.

\section{ACKNOWLEDGMENT}

The authors acknowledge the support to this paper by grant 2011/23217-0, São Paulo Research Foundation (FAPESP).

\section{References}

[1] R.A. Gupta, M.Y. Chow, "Networked Control System: Overview and Research Trends". IEEE Trans. on Industrial Electronics, vol. 57, n. 7, pp. 2527-2535, July, 2010. erosset

[2] J.R. Moyne, D.M. Tilbury, "The Emergence of Industrial Control Networks for Manufacturing Control, Diagnostics, and Safety Data". IEEE Tech. of Networked Control Systems, vol. 95, pp. 29-47, 2007. trosset

[3] J. Baillieul, P.J. Antsaklis, "Control and Communication Challenges in Networked Real Time Systems", IEEE Tech. of Networked Control Systems, Vol. 95, No. 1, pp. 09-28, 2007. trosset

[4] E.P. Godoy, A.J.V. Porto and R.Y. Inamasu, "Applied Simulation to Evaluate the Quality of Control of Networked Control Systems". in: Proc. of the 2010 IEEE Int. Conference On Networking, Sensing, and Control (ICNSC), Chicago, IL, USA, April 11-13, 2010. Crosset

[5] F.L. Lian, J.R. Moyne and D.M. Tilbury, "Network Design Consideration for Distributed Control Systems", IEEE Trans. on Control Systems Technology, vol. 10(2), pp. 297-307, 2002. Erosset

[6] C. Fischione, P. Park, P.Di Marco and K.H. Johansson, "Design Principles of Wireless Sensor Networks Protocols for Control
Applications", S.K. Mazumder (ed.), Wireless Networking Based Control, Ch. 9, Springer, pp. 203-238, 2011. Erosset

[7] M. Paavola, K. Leiviska, "Wireless Sensor Networks in Industrial Automation", J.S. Blanes (ed.), Factory Automation, Ch 10, InTech, pp. 201-220, 2010. frosset

[8] D.M. Anand, J.R. Moyne and D.M. Tilbury, "Performance evaluation of wireless networks for factory automation applications", in: Proc. Of the 5th Annual IEEE Conf. on Automation Science and Engineering (CASE), Bangalore, India, pp. 340-346, August 22-25, 2009. Erosset

[9] J.P Hespanha, P. Naghshtabrizi and Y. XU, "A Survey of Recent Results in Networked Control Systems", IEEE Technology of Networked Control Systems, vol. 95, no. 1, pp. 138-162, 2007. trosset

[10] P. Naghshtabrizi, J. Hespanha, "Implementation Considerations For Wireless Networked Control Systems", in: S.K. Mazumder (ed.), Wireless Networking Based Control, Ch 1, Springer, pp. 1-28, 2011. trosset

[11] E.P. Godoy, R.V. Sousa, A.J.V. Porto and R.Y. Inamasu, "Design of CAN-Based Distributed Control Systems with Optimized Configuration", Journal of the Brazilian Society of Mechanical Sciences and Engineering, vol. 32, n. 4, pp. 420-426, 2010. krosset

[12] E.P. Godoy, R.R.D. Pereira, F. Scorzoni, A.J.V. Porto and R.Y. Inamasu, "CAN-based Platform for the Study and Experimentation on Networked Control Systems (NCS)". in: Proc. of the 5th IFAC Symp. on Mechatronic Systems, Cambridge, MA, USA, pp.652-659, September 13-15, 2010. trosset

[13] E.P. Godoy, T.A Oliveira, I.S. Diniz and A.J.V. Porto, "A Testbed for Wireless Networked Control Systems based on CAN and ZigBee". in: Proc. of the 22nd International Congress of Mechanical Engineering (COBEM 2013), 2013, Ribeirão Preto. São Paulo, Brazil.

[14] CIA. CAN in Automation Website. Available in: < http://www.cancia.org >. Accessed in: September, 2011.

[15] K.H Johansson, M. Torngren and L. Nielsen, "Vehicle applications of controller area network", D. Hristu-Varsakelis, W.L. Levine (eds.), Handbook of Networked and Embedded Control Systems, Ch. 6, Birkhäuser, pp.741-766, 2005.

[16] B. Bernhardsson, J. Eker and J. Persson, "Bluetooth in Control", D. Hristu-Varsakelis, W.L. Levine (eds.), Handbook of Networked and Embedded Control Systems, Ch. 5, Birkhäuser, pp. 699-720, 2005. rosset

[17] P. Baronti, P. Pillai, V.W.C. Chook, V. Chessa, A. Gotta and Y.F. Hu, "Wireless sensor networks: A survey on the state of the art and the 802.15.4 and ZigBee standards". Computer Communications, vol. 30, no. 7, pp. 1655-1695, 2007. Erosset

[18] E.P. Godoy, F. Scorzoni, and A.J.V. Porto, "Evaluating serial ZigBee devices for application in wireless networked control systems. in: Proc. of the 38th Annual Conference of IEEE Industrial Electronics Society (IECON 2012), 2012, Montreal. Canada, p. 3176-3181. trosset

[19] E.P. Godoy, F. Scorzoni, G.T. Tangerino, A.J.V. Porto and R.Y. Inamasu, "Using Serial Bluetooth Converters as a Sensor Link in Networked Control Systems". in: Proc. of the 9th IEEE International Conference on Control \& Automation (IEEE ICCA 2001), 2011, Santiago, Chile. p. 461-466. Erostet

[20] K. Liu, Performance Evaluation of ZigBee Network for Embedded Electricity Meters. Master Thesis. Dept. of Elect. Eng., KTH Royal Inst. of Tech., Sweden, 63p, 2009.

[21] L. Eriksson, PID Controller Design and Tuning in Networked Control Systems, Doctorate Thesis, Dept. of Aut. and Systems Tech., Helsinki Univ. of Tech., Finland, 118p, 2008. 